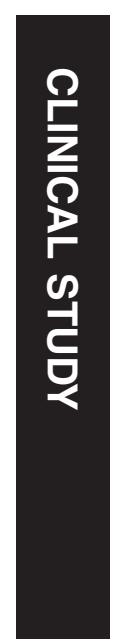

\section{Postnatal growth of the human optic nerve}

SL Bernstein ${ }^{1,2}$, M Meister $^{1}$, J Zhuo ${ }^{3}$ and RP Gullapalli ${ }^{3}$

\begin{abstract}
Purpose Although the length of the average human adult optic nerve $(\mathrm{ON})$ is known, the average length of the normal full-term, newborn $\mathrm{ON}$ has never been adequately evaluated, nor has the in vivo growth rate of the human $\mathrm{ON}$ been determined. We wanted to identify both the average length of the newborn human $\mathrm{ON}$ and its rate of anteroposterior growth.

Patients and methods Using MRIs from a newly generated set of normal newborn infants rescanned at 1 year, and from different aged groups, we calculated average newborn $\mathrm{ON}$ length and growth rate. Results The newborn human $\mathrm{ON}$ is $25.3 \pm 0.3 \mathrm{~mm}$ in length from globe to chiasm, and grows by $80 \%$ in length after birth, with maximum speed of elongation occurring in the first 3 years of life, attaining full length by 15 years of age.

Conclusion The human ON grows dramatically in the first 3 years of life, and continues to grow for the first two decades. These data are relevant for pediatric treatments that may impede or alter orbital growth in infants, and maximal susceptibility to oncological procedures in early childhood.

Eye (2016) 30, 1378-1380; doi:10.1038/eye.2016.141; published online 15 July 2016
\end{abstract}

Correspondence: SL Bernstein, Department of Ophthalmology and Visual Sciences, Lab of Molecular Research, University of Maryland School of Medicine, MSTF 5-77B, 10S. Pine Street, Baltimore, MD 21201, USA Tel: +1 410706 3712; Fax: +1 4107067057 . E-mail: sbernstein@som. umaryland.edu

Received: 22 December 2015

Accepted in revised form: 7 May 2016 Published online: 15 July 2016
Subjects and methods

\section{Human material}

All studies using human material were approved by the University of MarylandBaltimore institutional review board (IRB) prior to beginning the work.

A 'standard set' of magnetic resonance images (MRI-T1- and -T2-weighted) from normal full-term infants who were re-imaged at 1 year of age has recently become available for evaluation of growth trajectory through the infant brain imaging study (IBIS) network (www.ibisnetwork.org). Twelve identitymasked individuals from this set (six male and six female) were evaluated at both ages. We also included MRI-T1 and -T2 data from additional groups of masked Caucasian individuals at 3, 5, 10,15 , and 20 years of age. Data from these individuals were obtained from the University of Maryland-Baltimore radiographic center. Individuals were excluded from analysis if they had presented with any ocular or visual complaints. A minimum of 14 individuals were used for each age group. Male to female ratios were equal, except for the 10 -year $(46.7 \% ; n=15)$ and 15 -year $(42.9 \% ; n=14)$ groups.

\section{Analysis}

We utilized axial sections from good quality MRI scans. The ONs were measured bilaterally from the posterior of the globe to the middle of the optic chiasm on both T1- and T2-weighted images using the Leonardo MR workstation (Siemens Medical Solutions, Malvern, NY, USA) and the results were averaged. Furthermore, ON size was averaged for both sides from all individuals, with mean length in $\mathrm{mm}+/-\mathrm{SD}$ reported.

\section{Results}

MRI analysis revealed reduced ON myelin signal in full-term newborns, with progressive increase in the relative $\mathrm{T} 1 / \mathrm{T} 2$ signal intensity 

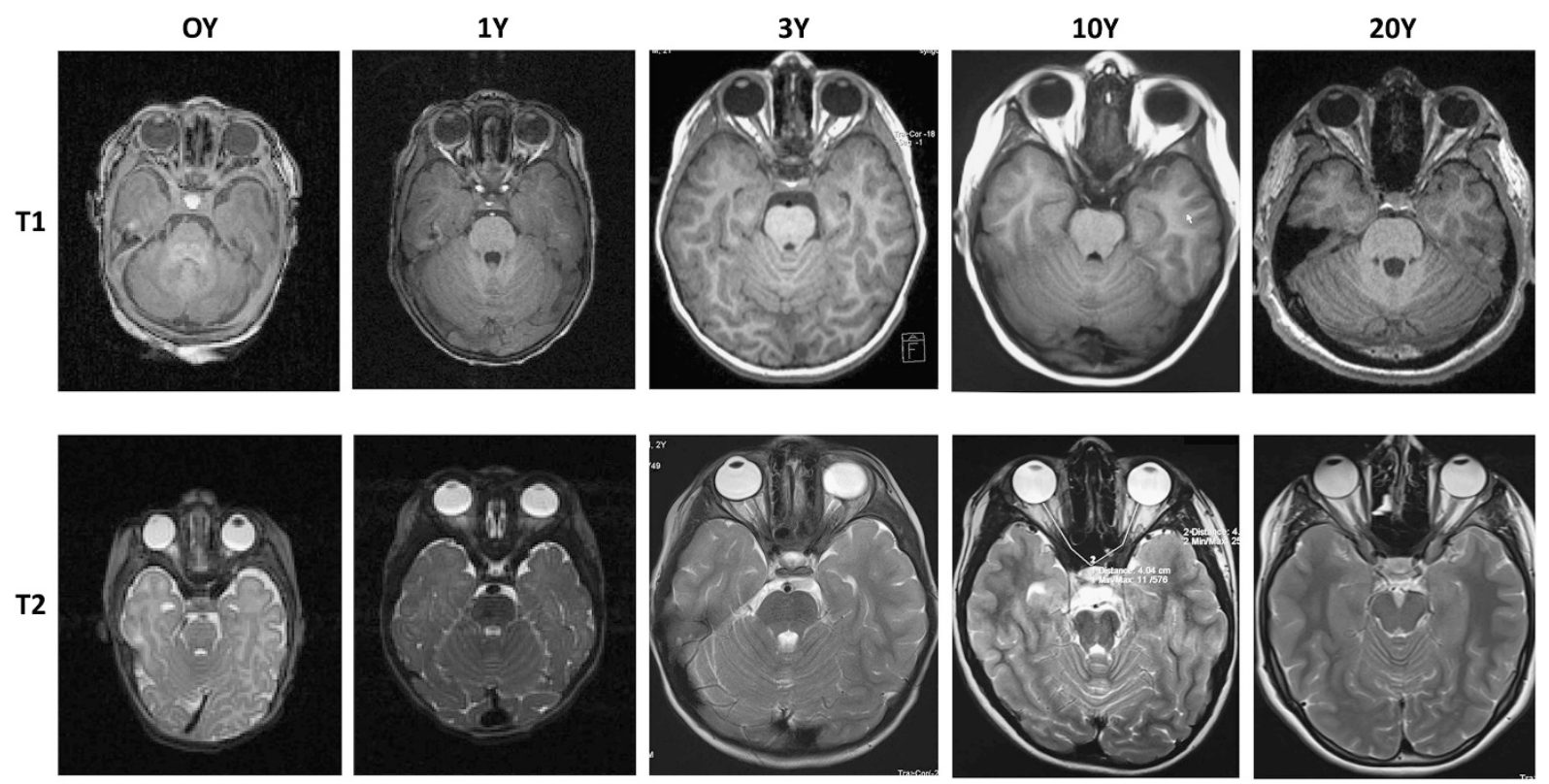

Figure 1 T1- and T2-weighted MRI-ON images at $0 \mathrm{Y}$, and at 1, 3, 5, and 20Y. 0, 1Y: Representative IBIS images (1/55 section total through entire brain). 3-20Y: Representative non-IBIS images (1/255 sections). ON measurement analysis is shown in the T2/10Y image. ON length was measured from the distal end of the widest A-P globe diameter, and the line extended using the Siemens Leonardo workstation through multiple scans to the anterior edge of the optic chiasm. Results from T1 and T2 images were averaged for each individual. Measurement from a single T2-weighted image is shown for a $10 \mathrm{Y}$ individual.

between newborns and 3 y/o (Figure 1; compare 0 and 3Y). T1/T2 signal intensity stabilized by 3 years of age. These changes are consistent with previously reported developmental increases in $\mathrm{ON}$ myelin density. ${ }^{4}$

The mean newborn $\mathrm{ON}$ is $25.3+/-\mathrm{mm}$ in length, reaching 45.3 (adult) by 20 years of age. Human ON growth is linear in the first 3 years of life, reaching $86 \%$ of the total adult length by this time (Figure 2; area indicated by (1): slope $\left.y=4.55 x+24.214 ; r^{2}=0.9991\right)$. Thereafter, the ON continues to grow slowly, until puberty at approximately 15 y/o (Figure 2; area indicated by (2): slope $\left.y=0.52 x+36.7 ; r^{2}=0.8214\right)$. ON length is stable through 20 years (Figure 2), indicating cessation of A-P growth.

\section{Discussion}

The current study is the first survey of total ON growth in vivo from birth to maturity. Our data reveal that the human $\mathrm{ON}$ grows by $80 \%$ after birth, with the greatest linear growth occurring within the first 3 years of postnatal life. ON growth continues at a slower rate from 5 to 15 years and then stops. This growth pattern likely corresponds to the increase in skull size around the age of puberty, and final skull growth.

There are a number of caveats that should be addressed. Ethnic self-reporting may minimize potential differences between races, and different races may have

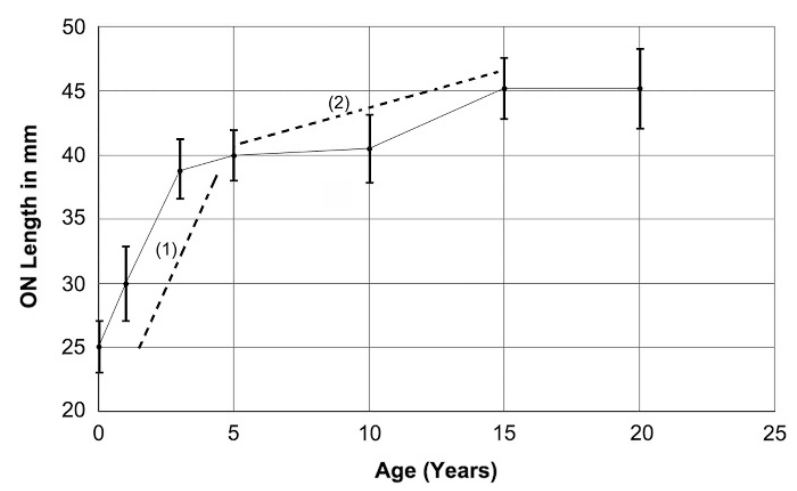

Figure 2 Postnatal ON growth analysis. ON length is shown on the ordinate, while age is shown on the abscissa. ON Mean size in $\mathrm{mm} \pm \mathrm{SD}$. ON growth is most rapid in the first 3 years of life, which then slows considerably from 5 to 10 years (compare slope (1) with slope (2)). A modest further increase in ON growth speed occurs between 10 and 15y, with final $\mathrm{ON}$ length achieved by 15 years.

slightly different growth patterns that were not evaluated in the present study. In addition, nearly equivalent numbers of both male and female patients were averaged, minimizing potential sex-associated differences in $\mathrm{ON}$ growth that may be present.

The ON early intensive growth period suggests that the axons in the juvenile ON may have enhanced regenerative capacity. Interestingly, it is still 
unknown whether postnatal ON axonal growth occurs as a diffuse phenomenon, or whether there is a specific focal site (for example, at the junction of the eye and $\mathrm{ON}) .^{5}$

Because the majority of $\mathrm{ON}$ growth occurs by 3 years of age, this postnatal interval likely represents a period of increased sensitivity and potential damage. Conversely, the factors associated with postnatal growth of the human $\mathrm{ON}$ may also increase early resistance and recovery to $\mathrm{ON}$ stressors such as elevated IOP, compared with treatment in adulthood. Understanding ON growth is an important step in evaluating diseases likely to affect visual function in the developing infant.

\section{Summary}

What was known before

- The human optic nerve (ON) grows after birth, but neither the length of the $\mathrm{ON}$ in living full-term infants nor the speed of nerve growth have been adequately documented.

What this study adds

- Using the recently completed MRI study of normal infant development and grouped studies from different aged individuals, the authors measured both the size of the normal infant $\mathrm{ON}$ and its average growth from birth to adulthood.

- The human ON grows $80 \%$ in the first 3 years of life, reaching adult length by 15 years of age.

\section{Conflict of interest}

The authors declare no conflict of interest.

\section{Author contributions}

SLB conceived of and designed the study, and wrote the majority of the manuscript. MM identified the patients in the University database, performed many of the individual analyses and helped write the manuscript. JZ evaluated the data and helped with the measurement transformations, selected appropriate individual images for analysis. RPG contributed the data from the IBIS dataset, helped interpret the data, suggested the appropriate measurement tools and helped write the manuscript.

\section{Acknowledgements}

This study was funded by NEI grant NEI EY019529 (to SLB) and grant 1S10RR019214 (to RPG). The excellent technical assistance of Ms. Erma Owen is gratefully acknowledged.

\section{References}

1 Scammon RE, Armstrong EL. On the growth of the human eyeball and optic nerve. J Comp Neurol 1925; 38: 165-219.

2 Miller NR. Embryology of the afferent visual system. In: Walsh and Hoyt's Clinical Neuro-Ophthalmology, Vol 1, 4th edn. Williams and Wilkins: Baltimore, MD, USA, 1982, pp 3-10.

3 Morriss-Kay GM, Wilkie AO. Growth of the normal skull vault and its alteration in craniosynostosis: insights from human genetics and experimental studies. J Anat 2005; 207: 637-653.

4 Geng X, Gouttard S, Sharma A, Gu H, Styner M, Lin W et al. Quantitative tract-based white matter development from birth to age 2 years. Neuroimage 2012; 61: 542-557.

5 Goldberg JL. How does an axon grow? Genes Dev 2003; 17: 941-958. 